\title{
Can Oscillatory Alpha-Gamma Phase-Amplitude Coupling be Used to Understand and Enhance TMS Effects?
}

\author{
Johanna Wagner $^{1 *}$, Scott Makeig ${ }^{1}$, David Hoopes ${ }^{2}$ and Mateusz Gola ${ }^{1,3}$ \\ ${ }^{1}$ Swartz Center for Computational Neurosciences, Institute for Neural Computation, University of California, San Diego, \\ San Diego, CA, United States, ²Department of Radiation Medicine and Applied Sciences, School of Medicine, \\ University of California, San Diego, San Diego, CA, United States, ${ }^{3}$ Institute of Psychology, Polish Academy of Sciences, \\ Warsaw, Poland
}

Recent applications of simultaneous scalp electroencephalography (EEG) and transcranial magnetic stimulation (TMS) suggest that adapting stimulation to underlying brain states may enhance neuroplastic effects of TMS. It is often assumed that longerlasting effects of TMS on brain function may be mediated by phasic interactions between TMS pulses and endogenous cortical oscillatory dynamics. The mechanisms by which TMS exerts its neuromodulatory effects, however, remain unknown. Here, we discuss evidence concerning the functional effects on synaptic plasticity of oscillatory crossfrequency coupling in cortical networks as a potential framework for understanding the neuromodulatory effects of TMS. We first discuss evidence for interactions between endogenous oscillatory brain dynamics and externally induced electromagnetic field

OPEN ACCESS

Edited by: Filippo Brighina, University of Palermo, Italy

Reviewed by:

Mitchell Ryan Goldsworthy, University of Adelaide, Australia

Domenica Veniero, University of Glasgow, United Kingdom

${ }^{*}$ Correspondence: Johanna Wagner j9wagner@ucsd.edu

Received: 02 May 2019 Accepted: 12 July 2019 Published: 31 July 2019

Citation:

Wagner J, Makeig S, Hoopes D and Gola M (2019) Can Oscillatory

Alpha-Gamma Phase-Amplitude Coupling be Used to Understand and Enhance TMS Effects? Front. Hum. Neurosci. 13:263. doi: 10.3389/fnhum.2019.00263 activity. Alpha band (8-12 Hz) activities are of special interest here because of the wide application and therapeutic effectiveness of rhythmic TMS (rTMS) using a stimulus repetition frequency at or near $10 \mathrm{~Hz}$. We discuss the large body of literature on alpha oscillations suggesting that alpha oscillatory cycles produce periodic inhibition or excitation of neuronal processing through phase-amplitude coupling (PAC) of low-frequency oscillations with high-frequency broadband (or gamma) bursting. Such alpha-gamma coupling may reflect excitability of neuronal ensembles underlying neuroplasticity effects of TMS. We propose that TMS delivery with simultaneous EEG recording and near real-time estimation of source-resolved alpha-gamma PAC might be used to select the precise timing of TMS pulse deliveries so as to enhance the neuroplastic effects of TMS therapies.

Keywords: transcranial magnetic stimulation, TMS, phase-amplitude coupling, PAC, neurostimulation, oscillations, EEG

\section{INTRODUCTION}

Non-invasive transcranial magnetic stimulation (TMS) of the human brain has gained increasing popularity over the last decades and today is being widely used in both research and clinical applications. In TMS, brief, high-intensity electromagnetic pulses are produced in one or more wire coils (transducers) placed tangential to the scalp, inducing electrical currents 
in the underlying brain area. TMS can be applied as single, isolated pulses or as trains of stimuli [termed repetitive or rhythmic TMS (rTMS)], producing effects on the brain that can accumulate with repeated exposure and outlast the course of treatment (Rossi and Rossini, 2004; Ridding and Rothwell, 2007; Thut and Pascual-Leone, 2010). The effects of rTMS on brain activity can be observed near the site of maximal cortical stimulation as well as at anatomically remote but functionally connected cortical and subcortical areas (Strafella et al., 2001; Pogarell et al., 2006; Tik et al., 2017) suggesting that rTMS may modulate the dynamics of affected brain circuits (Medaglia et al., 2017; Vöröslakos et al., 2018). Possible long-lasting neuromodulatory effects of rTMS on brain circuits are of great interest in the clinical therapeutic arena, as they are thought to have potential benefit for a wide range of neurological and psychiatric pathologies thought to be characterized by disturbance in functional connectivity among brain regions (for reviews, see Schnitzler and Gross, 2005; Uhlhaas and Singer, 2010).

The precise mechanisms by which TMS exerts its neuromodulatory effects remain unknown. Research in humans suggests that both immediate and longer-term effects of rTMS are mediated by the interaction of the induced electrical current with endogenous oscillatory dynamics (Klimesch et al., 2003; Thut et al., 2011). These studies show that targeting subject- and task-specific oscillatory frequency bands can increase the effects on oscillatory band power as measured by scalp electroencephalography (EEG) and also, subsequent cognitive and behavioral performance (Klimesch et al., 2003; Thut et al., 2011; Veniero et al., 2011; for reviews, see Bergmann et al., 2016; Thut et al., 2017; Hanslmayr et al., 2019). Timing TMS pulse presentations to specific phases of ongoing oscillatory activity has also been demonstrated to increase subsequent corticospinal excitability-a measure of cortical plasticity (Bergmann et al., 2012; Zrenner et al., 2018). This suggests that electric field activity in the brain produced by TMS pulses may enhance underlying cortical excitability which is modulated by oscillatory field activity occurring within distributed brain networks, thereby affecting basic synaptic mechanisms producing long-term potentiation (LTP) and/or depression (LTD) within those networks (Ridding and Rothwell, 2007).

Here, we first review evidence for interactions between externally induced brain electric field potentials and endogenous cortical field activity. We then discuss how high and low excitability states relate to the phase of oscillatory field potentials, and how phase controls excitability states through cross-frequency coupling. Based on this body of research we argue that brain stimulation timed to particular phases of spontaneous low-frequency oscillations may enhance neural excitability, by increasing occurrence of appropriately timed high-frequency gamma oscillations through the mechanism of cross-frequency phase-amplitude coupling (PAC). The neurophysiological properties of oscillatory coupling may explain oscillatory-phase-guided rTMS neuroplasticity effects and thereby help to identify high-excitability phases to best target with TMS.

\section{BASIC UNDERSTANDING OF INTERACTIONS BETWEEN ENDOGENOUS OSCILLATIONS AND EXTERNALLY INDUCED BRAIN ELECTRIC FIELD ACTIVITY}

Direct evidence for interaction between externally-induced brain electric field activity and endogenous cortical oscillations comes from recordings of local field potentials and multiunit activity in animals. In vitro recordings in animal brain slices have shown that applied weak oscillatory electric fields affect the transmembrane voltage of nearby neurons, biasing neuronal spike timing (Anastassiou et al., 2011; Anastassiou and Koch, 2014). Fröhlich and McCormick (2010) induced weak sinusoidal currents in vitro leading to concentrated bursts of neural firing in affected neuropile in the applied current low-frequency oscillation pattern. This occurred for levels of induced current well below those needed to increase the net firing rate of the involved neurons, but comparable to levels of in vivo endogenous local field potential in the same tissues.

Results of Ali et al. (2013) suggest that matching stimulation frequency to endogenous brain activity is a crucial requirement for weak oscillatory electric fields to have an effect on network dynamics since the depolarization caused by a weak supplied electric field is too small to activate neurons at rest. Weak electric fields applied at the endogenous oscillation frequency may enhance endogenous oscillations but fail to induce a frequency shift when the stimulation frequency is not matched to the endogenous oscillation (Schmidt et al., 2014). In essence, neurons need to be close to their firing threshold for a stimulation-induced sub-millivolt perturbation in membrane voltage to effectively modulate endogenous network neural spiking statistics and affect brain network dynamics.

Research in humans has shown that rTMS tuned to endogenous EEG oscillations enhances cortical oscillations in the targeted band and may also produce behavioral changes (Sauseng et al., 2009b; Romei et al., 2010). Thut et al. (2011), for example, reported phase-locking of EEG activity to magnetic pulse trains of participant- and task-specific alpha-frequency rTMS. Entrained rTMS-evoked EEG activity may also outlast the stimulation, suggesting that an endogenous, rTMS-induced mode of brain activity has been produced by the stimulation (Hanslmayr et al., 2014). As reported by Klimesch et al. (2003), increased alpha power following rTMS pulse trains delivered at a subject's individual alpha frequency was associated with a significant improvement in subsequent performance of a mental rotation task.

Other research supports the concept that ongoing oscillations create periodic "windows of excitability" that can be targeted with TMS. Dugué et al. (2011), for example, showed that the phase of ongoing $(8-12 \mathrm{~Hz})$ alpha oscillations, within the $400 \mathrm{~ms}$ before a TMS pulse applied over visual cortex, significantly co-varied with the pulse-induced visual illusions (phosphenes). Similar observations have been described in the sensorimotor system for which the most dominant oscillatory frequency is the $(8-12 \mathrm{~Hz})$ mu rhythm. Zrenner et al. (2018) triggered TMS 
pulse triplets (three pulses at $100 \mathrm{~Hz}$ ) at varying intervals (longer than $0.75 \mathrm{~s}$ ) targeted to occur at negative or positive peaks of healthy participants' spontaneous EEG mu rhythms. Only stimulation at the surface negative peak of the mu-rhythm cycles resulted in a LTP like increase in corticospinal excitability [as measured by subsequent increase of the motor-evoked potential (MEP) amplitude]. Bergmann et al. (2012) triggered single-pulse TMS over the primary motor cortical hand area within EEG relative (surface-negative) "up-states" and (surface-positive) "down-states" during sleep. Both TMS-evoked and subsequent MEPs were consistently larger when stimulation occurred during slow oscillatory (negative-going) up-states than during (surfacepositive) down-states. These results can be explained by direct effects of local field activity on neural excitability, including little understood ephaptic (non-synaptic) effects on the intra-neuronal environment (Fröhlich and McCormick, 2010; Anastassiou et al., 2011; Anastassiou and Koch, 2014). This work raises the intriguing possibility that real-time information on current brain state derived from EEG recording can be used to maximize TMS induction of cortical plasticity in humans.

\section{EVIDENCE FOR CORTICAL EXCITABILITY STATES CHANGES WITH ALPHA OSCILLATORY CYCLES}

Understanding how cortical excitability is affected by endogenous local field potentials, therefore, seems crucial to further development and optimization of TMS stimulation protocols. As outlined above, oscillations in local cortical field potentials are now seen to both reflect and induce cyclical variation in the excitability of involved cortical neuronal ensembles (Bishop, 1933; Freeman and Rogers, 2002; Vanhatalo et al., 2004), making them more likely to fire in one phase of the cycle than in another (Klausberger et al., 2003; Haider and McCormick, 2009; Canolty and Knight, 2010; Canolty et al., 2010). Targeting oscillations in the $(8-12 \mathrm{~Hz})$ alpha frequency band is of special interest, as most current clinical TMS protocols involve some form of stimulation in this frequency range. For example, $10-\mathrm{Hz}$ rTMS over frontal brain areas has proven to have therapeutic benefit in treatment-resistant depression; accordingly, most rTMS protocols approved to date by the United States Food and Drug Administration (FDA) involve 10-Hz stimulation (O'Reardon et al., 2007; George et al., 2010; Perera et al., 2016).

There is growing evidence that $8-12 \mathrm{~Hz}$ posterior alpha and sensorimotor $(\mathrm{mu})$ oscillations play a significant role in modulating brain information processing in humans by providing a periodic inhibitory influence within their generator regions (Klimesch et al., 2007; Jensen and Mazaheri, 2010; Mathewson et al., 2011). Recent findings suggest that mu rhythms exercise strong inhibitory influence on local neuronal spike timing firing rate. Haegens et al. (2011) reported a rhythmic relation between mu-rhythm oscillations in monkey sensorimotor cortex and neuronal spiking, with neuronal firing highest at the (surface-negative) trough of the mu-rhythm cycle. Ai and Ro (2014) demonstrated that humans' ability to perceive a weak tactile stimulus was predicted by the mu phase angle at stimulus onset in the EEG, suggesting that sensorimotor mu rhythms wield a strong inhibitory control on tactile perception.

A similar relationship seems to hold for alpha oscillations in the visual cortex. Mercier et al. (2015) for example, showed using ECoG data that reaction times are faster when local auditory and visual cortical theta/low alpha rhythms $(5-8 \mathrm{~Hz})$ are both in phase with the onset of an audiovisual stimulus. Other studies demonstrated that both phase and power of pre-stimulus alpha oscillations affect visual detection (van Dijk et al., 2008; Busch et al., 2009; Mathewson et al., 2009). Visual discrimination ability decreases with an increase in pre-stimulus alpha power (van Dijk et al., 2008) while detection performance for attended stimuli fluctuates in time with the pre-stimulus phase of spontaneous alpha oscillations (Busch and VanRullen, 2010). This phasic modulation of detection performance increases with stronger alpha entrainment to a rhythmic stimulus presentation (Spaak et al., 2014). Other research demonstrates that the phase of EEG alpha rhythm over posterior brain regions can reliably predict both stimulus-elicited cortical activation levels and subsequent visual detection (Mathewson et al., 2009). As well, blood oxygenation-level-dependent (BOLD) responses to brief fixation events have also been shown to vary as a function of the alpha phase of EEG independent component effective source processes (Scheeringa et al., 2011).

Research also shows that alpha oscillations influence the temporal resolution of perception. Two briefly presented visual stimuli may be perceived as a single stimulus or as two separate stimuli depending on whether they fall in one or two separate alpha cycles depending on the frequency of the alpha oscillation (Samaha and Postle, 2015). These and related findings (Varela et al., 1981; Zauner et al., 2012) have led to the conclusion that the frequency of the alpha cycle indexes the duration of "perceptual windows" (e.g., during the surface-negative phase of the alpha cycle), and controls variation in both the sensitivity and temporal resolution of visual perception (for reviews, see Hanslmayr et al., 2011; Mathewson et al., 2011; Shapiro et al., 2017).

Note that the direction of the relationship between cortical surface negative and positive peaks is ambiguous for sulcal rhythms since the polarity of alpha negative/positive peaks depends on the orientation of the vortical source patch in relation to the cortical surface. In addition, many of the above cited studies have analyzed EEG channel data (Mathewson et al., 2009; Busch and VanRullen, 2010; Samaha and Postle, 2015; Zrenner et al., 2018) which adds additional ambiguity in terms of oscillatory phase. By the broad spread of brain volume conduction, each EEG channel signal sums potentials from many effective brain sources (for example, see Makeig et al., 2004; Onton et al., 2005, 2006; Brunner et al., 2016). This needs to be considered when targeting alpha negative/positive peaks with TMS. Source-resolved estimation of EEG oscillatory phase, for example using Independent Component Analysis (Bell and Sejnowski, 1995; Makeig et al., 1996), can help to resolve this ambiguity.

Nevertheless, the here discussed studies suggest that mu and alpha rhythm cycles constrain neural spikes into occurring during brief time windows, leading to periodic suppression of 
neural processing with cortical surface negative and positive peaks in the mu/alpha cycle representing high and low excitability states respectively. Zrenner et al. (2018) provide deterministic evidence for this long-held belief, proposed by Elbert and Rockstroh (1987).

\section{RELATIONSHIP TO CROSS-FREQUENCY PHASE-AMPLITUDE COUPLING OF NEURONAL OSCILLATIONS}

What are the underlying functional mechanisms by which oscillatory phase changes the excitability of the local cortical area and state? Nested hierarchical cross-frequency PAC of cortical potentials, wherein phase in lower frequency bands modulates amplitude in respectively higher bands, has been proposed as a general mechanism supporting the encoding, storage, and retrieval of information in neural networks (Schroeder and Lakatos, 2009; Canolty and Knight, 2010; Fell and Axmacher, 2011; Bergmann and Born, 2018; Reinhart and Nguyen, 2019). Slow oscillations consist of alternating states of synchronized depolarization (up-state) and hyperpolarization (down-state) that propagate throughout the cortex, also reaching the thalamus via cortico-thalamic projections. Note that cortico-thalamic feedback may play a key role in the temporal control of cortical excitability by mediating phase alignment of neuronal firing and slow oscillatory peak depolarization.

The most-studied example of PAC is theta-gamma PAC in the hippocampus and cortex during working memory, information encoding, and retrieval (Fell and Axmacher, 2011) that is linked to theta phase-dependent processes of synaptic potentiation and depotentiation (Huerta and Lisman, 1995). It is hereby assumed that the phase of these spontaneous low-frequency oscillations control the excitability of local cortical neuronal ensembles, making them more likely to fire (Klausberger et al., 2003; Haider and McCormick, 2009; Canolty and Knight, 2010). This results in a systematic enhancement of responses to events occurring during high-excitability phases concurrent with broadband $(30-200 \mathrm{~Hz})$ gamma oscillatory bursts in cortical recordings, and suppression of responses to events occurring during low-excitability phases (Large and Kolen, 1994; Lakatos et al., 2005, 2008). Broadband gamma (30-200 Hz) activity has been suggested to reflect and index local neuronal population activity (Miller et al., 2009, 2014) indicating a state of high neuronal excitability (Fries et al., 2007).

Studies have demonstrated that timing of gamma bursts in the EEG is commonly modulated by alpha phase (Osipova et al., 2008; Voytek et al., 2010; for a review, see Canolty and Knight, 2010). The alpha cycle supposedly acts here as periodic inhibition-gamma bursts occur only during the cortical surface-negative troughs of the alpha cycle, and when the amplitude of alpha oscillations is sufficiently low. The strength of this relationship may change with movement or other cortical activation states (see Figure 1). A recent study (Herring et al., 2019) has provided deterministic evidence for the modulation of stimulus-induced gamma-band oscillations through alpha oscillatory phase. The authors applied weak alternating currents at subject's individual alpha frequency $\pm 4 \mathrm{~Hz}$ to the occipital cortex to mimic the functional effects of periodic inhibition during spontaneous alpha oscillations. The

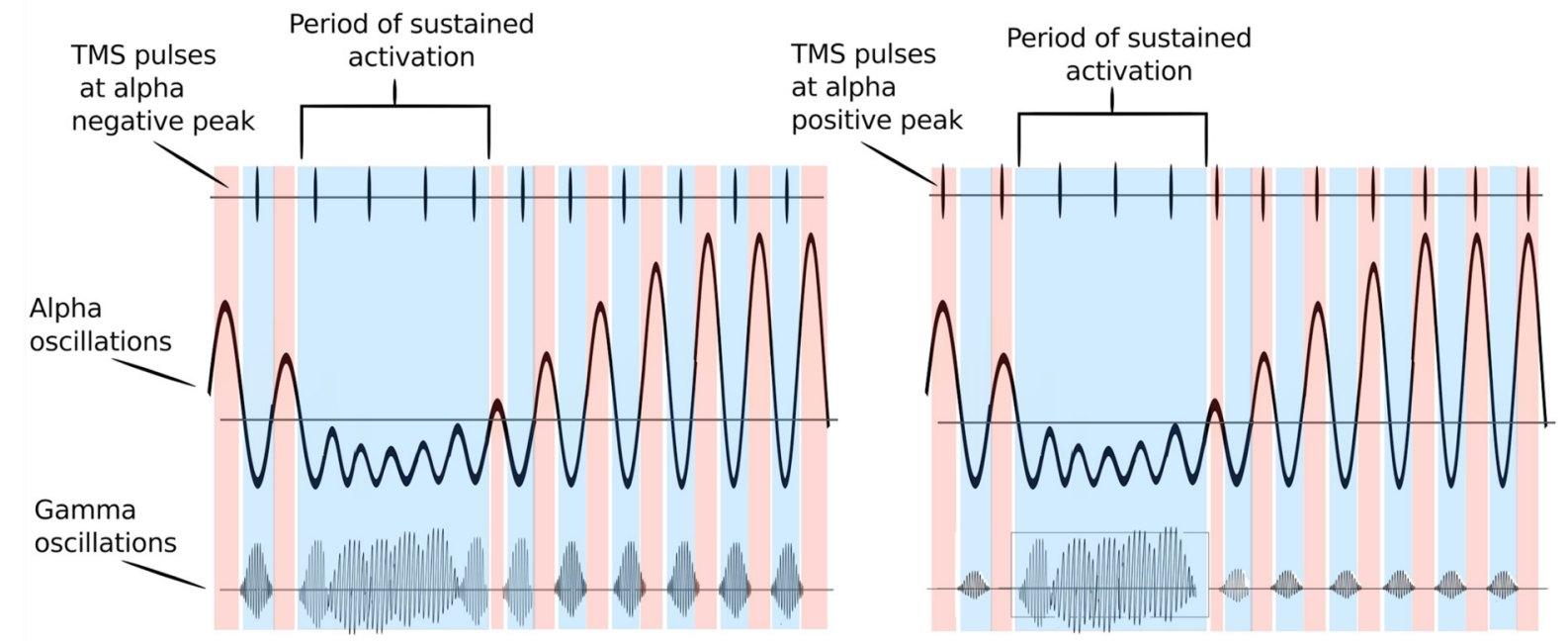

FIGURE 1 | Possible mechanism of how alpha oscillations act on gating neural excitability: red color periods indicate periods of inhibition (alpha positive peak), while blue color indicates periods of activation (alpha trough). Transcranial magnetic stimulation (TMS) pulses are represented by vertical lines in the upper part of the figure. The bursts of gamma at each alpha cycle trough represent windows of neuronal processing. Left Panel: TMS pulses are delivered at alpha troughs. They thus coincide with gamma bursts that are coupled to alpha troughs, enhancing them. If TMS pulses arrive during high excitability phases at alpha troughs (blue colored periods), they occur simultaneously with gamma bursts and are able to enhance local brain processing. Right Panel: TMS pulses are delivered at alpha positive peaks. They thus occur at periods of relative inhibition when gamma bursts are absent and no enhancement of neural activity may occur. When alpha oscillations are sufficiently suppressed neurons can fire freely and TMS pulses delivered during this period can enhance gamma bursts irrespective of the phase of alpha oscillations. Adapted from Osipova et al. (2008) and Jensen and Mazaheri (2010). 
authors found that in fact the induced currents rhythmically suppressed visual stimulus-induced gamma-band power. The degree of gamma-band suppression predicted the reduction in visual detection performance, suggesting a direct modulation of cortical excitability by rhythmically shifting the neurons' membrane potential. The here outlined ideas are supported by research showing that $10-\mathrm{Hz}$ rTMS strengthened alpha-gamma cross-frequency phase synchrony and predicted changes in task accuracy in a visual working memory task (Hamidi et al., 2009).

Many EEG and ECoG studies show that a decrease in mu power in motor cortices is related to increased activation of the cortical area (Pfurtscheller et al., 1997; Crone et al., 1998; Pfurtscheller and Lopes da Silva, 1999; Miller et al., 2007). During movement as well as other activation states known to transiently block mu rhythm amplitude (Pfurtscheller and Neuper, 1997; Crone et al., 1998), alpha-gamma PAC may be diminished or eliminated (as also shown for beta-gamma coupling by Miller et al., 2007), and gamma bursts may occur freely throughout the alpha cycle. Other studies investigating the relationship between corticospinal excitability (as measured with MEPs) and alpha power showed that MEPs are larger when pre-stimulus mu power is lower (Zarkowski et al., 2006; Sauseng et al., 2009a), and pre-stimulus gamma power is higher (Zarkowski et al., 2006). Sauseng et al. (2009a) also showed that this effect was specific for local EEG alpha activity at sites overlying the cortical motor areas to which the TMS pulses were applied (as verified using source localization).

Thus, during a cortical activation state where alpha/mu power is suppressed, TMS pulses delivered at any phase of $\mathrm{mu} / \mathrm{alpha}$ cycles may increase neuronal firing thus increasing subsequently cortical excitability. Instead during periods of increased mu/alpha power TMS pulses may best be delivered during surface negative alpha troughs to increase cortical activation states to be most effective.

\section{CONCLUSION}

The studies discussed above suggest that mechanisms of PAC in local cortical brain field activities, the most prominent of which may dominate scalp EEG signals, could

\section{REFERENCES}

Ai, L., and Ro, T. (2014). The phase of prestimulus $\alpha$ oscillations affects tactile perception. J. Neurophysiol. 111, 1300-1307. doi: 10.1152/jn. 00125.2013

Ali, M. M., Sellers, K. K., and Fröhlich, F. (2013). Transcranial alternating current stimulation modulates large-scale cortical network activity by network resonance. J. Neurosci. 33, 11262-11275. doi: 10.1523/JNEUROSCI.586712.2013

Anastassiou, C. A., and Koch, C. (2014). Ephaptic coupling to endogenous electric field activity: why bother? Curr. Opin. Neurobiol. 31C, 95-103. doi: 10.1016/j. conb.2014.09.002

Anastassiou, C. A., Perin, R., Markram, H., and Koch, C. (2011). Ephaptic coupling of cortical neurons. Nat. Neurosci. 14, 217-223. doi: 10.1038/nn.2727

Bell, A. J., and Sejnowski, T. J. (1995). An information-maximization approach to blind separation and blind deconvolution. Neural Comput. 7, 1129-1159. doi: 10.1162/neco.1995.7.6.1129 be exploited as a tool for more efficient TMS stimulation by incorporating information on the timing of neuronal excitability states.

Clinical TMS therapy has not changed much over the last 30 years with similar treatment protocols applied across different patient groups and a variety of disorders. One of the main practical issues in TMS therapy is that TMS after-effects are notoriously inconsistent, the same stimulation protocol inducing neural plasticity effects in opposite directions (Müller-Dahlhaus et al., 2008; Ziemann and Siebner, 2015). Bergmann et al.'s (2012) and Zrenner et al.'s (2018) studies provide insight into how stimulation protocols can be improved by increasing neuroplasticity through timing TMS pulses to oscillatory high excitability phases. These results were obtained in the motor cortex, however high and low excitability oscillatory phases likely differ over brain areas and frequency bands. This raises the question of how we can reliably identify high excitability phases of oscillations to target with TMS. Estimation of PAC may help to determine which exact phase of a given oscillation in the target brain area has the highest excitability. Methods for the estimation of event-related and time-resolved PAC (Voytek et al., 2013; Martínez-Cancino et al., 2019) may be either implemented before TMS stimulation or integrated into a real-time system to adapt timing of TMS pulses. Real-time estimation of PAC during TMS stimulation might be used to index of neuroplasticity and help determine the efficiency of the stimulation or predict the success of TMS therapy.

\section{AUTHOR CONTRIBUTIONS}

JW, MG and SM developed the concept for the manuscript. JW did the literature research and drafted the manuscript. MG, SM and $\mathrm{DH}$ provided comments to improve the manuscript.

\section{FUNDING}

This work was supported by the University of California (UC) San Diego Foundation [2467 sponsor Kreutzkamp]; by gifts to UC San Diego from The Swartz Foundation (Sag Harbor, NY, USA), and by an anonymous donor.

Bergmann, T. O., and Born, J. (2018). Phase-amplitude coupling: a general mechanism for memory processing and synaptic plasticity? Neuron $97,10-13$. doi: 10.1016/j.neuron.2017.12.023

Bergmann, T. O., Karabanov, A., Hartwigsen, G., Thielscher, A., and Siebner, H. R. (2016). Combining non-invasive transcranial brain stimulation with neuroimaging and electrophysiology: current approaches and future perspectives. Neuroimage 140, 4-19. doi: 10.1016/j.neuroimage.2016. 02.012

Bergmann, T. O., Mölle, M., Schmidt, M. A., Lindner, C., Marshall, L., Born, J., et al. (2012). EEG-guided transcranial magnetic stimulation reveals rapid shifts in motor cortical excitability during the human sleep slow oscillation. J. Neurosci. 32, 243-253. doi: 10.1523/JNEUROSCI.4792-11.2012

Bishop, G. (1933). Cyclic changes in excitability of the optic pathway of the rabbit. Am. J. Physiol. 103, 213-224. doi: 10.1152/ajplegacy.1932.103.1.213

Brunner, C., Billinger, M., Seeber, M., Mullen, T. R., and Makeig, S. (2016). Volume conduction influences scalp-based connectivity estimates. Front. Comput. Neurosci. 10:121. doi: 10.3389/fncom.2016.00121 
Busch, N. A., and VanRullen, R. (2010). Spontaneous EEG oscillations reveal periodic sampling of visual attention. Proc. Natl. Acad. Sci. U S A 107, 16048-16053. doi: 10.1073/pnas.1004801107

Busch, N. A., Dubois, J., and VanRullen, R. (2009). The phase of ongoing EEG oscillations predicts visual perception. J. Neurosci. 29, 7869-7876. doi: 10.1523/JNEUROSCI.0113-09.2009

Canolty, R. T., Ganguly, K., Kennerley, S. W., Cadieu, C. F., Koepsell, K., Wallis, J. D., et al. (2010). Oscillatory phase coupling coordinates anatomically dispersed functional cell assemblies. Proc. Natl. Acad. Sci. U S A 107, 17356-17361. doi: 10.1073/pnas.1008306107

Canolty, R. T., and Knight, R. T. (2010). The functional role of cross-frequency coupling. Trends Cogn. Sci. 14, 506-515. doi: 10.1016/j.tics.2010.09.001

Crone, N. E., Miglioretti, D. L., Gordon, B., Sieracki, J. M., Wilson, M. T., Uematsu, S., et al. (1998). Functional mapping of human sensorimotor cortex with electrocorticographic spectral analysis. I. $\alpha$ and $\beta$ eventrelated desynchronization. Brain 121, 2271-2299. doi: 10.1093/brain/121. 12.2271

Dugué, L., Marque, P., and VanRullen, R. (2011). The phase of ongoing oscillations mediates the causal relation between brain excitation and visual perception. J. Neurosci. 31, 11889-11893. doi: 10.1523/JNEUROSCI.1161-11.2011

Elbert, T., and Rockstroh, B. (1987). Threshold regulation-a key to the understanding of the combined dynamics of EEG and event-related potentials. J. Psychophysiol. 1, 317-333.

Fell, J., and Axmacher, N. (2011). The role of phase synchronization in memory processes. Nat. Rev. Neurosci. 12, 105-118. doi: 10.1038/nrn2979

Freeman, W. J., and Rogers, L. J. (2002). Fine temporal resolution of analytic phase reveals episodic synchronization by state transitions in $\gamma$ EEGs. J. Neurophysiol. 87, 937-945. doi: 10.1152/jn.00254.2001

Fries, P., Nikolić, D., and Singer, W. (2007). The $\gamma$ cycle. Trends Neurosci. 30, 309-316. doi: 10.1016/j.tins.2007.05.005

Fröhlich, F., and McCormick, D. A. (2010). Endogenous electric fields may guide neocortical network activity. Neuron 67, 129-143. doi: 10.1016/j.neuron.2010. 06.005

George, M. S., Lisanby, S. H., Avery, D., McDonald, W. M., Durkalski, V., Pavlicova, M., et al. (2010). Daily left prefrontal transcranial magnetic stimulation therapy for major depressive disorder: a sham-controlled randomized trial. Arch. Gen. Psychiatry 67, 507-516. doi: 10.1001/archgenpsychiatry.2010.46

Haegens, S., Nácher, V., Luna, R., Romo, R., and Jensen, O. (2011). a-Oscillations in the monkey sensorimotor network influence discrimination performance by rhythmical inhibition of neuronal spiking. Proc. Natl. Acad. Sci. U S A 108, 19377-19382. doi: 10.1073/pnas.111719010

Haider, B., and McCormick, D. A. (2009). Rapid neocortical dynamics: cellular and network mechanisms. Neuron 62, 171-189. doi: 10.1016/j.neuron.2009. 04.008

Hamidi, M., Slagter, H. A., Tononi, G., and Postle, B. R. (2009). Repetitive transcranial magnetic stimulation affects behavior by biasing endogenous cortical oscillations. Front. Integr. Neurosci. 3:14. doi: 10.3389/neuro.07. 014.2009

Hanslmayr, S., Axmacher, N., and Inman, C. S. (2019). Modulating human memory via entrainment of brain oscillations. Trends Neurosci. 42, 485-499. doi: 10.1016/j.tins.2019.04.004

Hanslmayr, S., Gross, J., Klimesch, W., and Shapiro, K. L. (2011). The role of $\alpha$ oscillations in temporal attention. Brain Res. Rev. 67, 331-343. doi: 10.1016/j. brainresrev.2011.04.002

Hanslmayr, S., Matuschek, J., and Fellner, M. C. (2014). Entrainment of prefrontal $\beta$ oscillations induces an endogenous echo and impairs memory formation. Curr. Biol. 24, 904-909. doi: 10.1016/j.cub.2014.03.007

Herring, J. D., Esterer, S., Marshall, T. R., Jensen, O., and Bergmann, T. O. (2019). Low-frequency alternating current stimulation rhythmically suppresses $\gamma$-band oscillations and impairs perceptual performance. Neuroimage 184, 440-449. doi: 10.1016/j.neuroimage.2018.09.047

Huerta, P. T., and Lisman, J. E. (1995). Bidirectional synaptic plasticity induced by a single burst during cholinergic theta oscillation in CA1 in vitro. Neuron 15 , 1053-1063. doi: 10.1016/0896-6273(95)90094-2

Jensen, O., and Mazaheri, A. (2010). Shaping functional architecture by oscillatory $\alpha$ activity: gating by inhibition. Front. Hum. Neurosci. 4:186. doi: $10.3389 /$ fnhum.2010.00186
Klausberger, T., Magill, P. J., Márton, L. F., Roberts, J. D. B., Cobden, P. M., Buzsáki, G., et al. (2003). Brain-state-and cell-type-specific firing of hippocampal interneurons in vivo. Nature 421, 844-848. doi: 10.1038/ nature 01374

Klimesch, W., Sauseng, P., and Gerloff, C. (2003). Enhancing cognitive performance with repetitive transcranial magnetic stimulation at human individual $\alpha$ frequency. Eur. J. Neurosci. 17, 1129-1133. doi: 10.1046/j.14609568.2003.02517.x

Klimesch, W., Sauseng, P., and Hanslmayr, S. (2007). EEG $\alpha$ oscillations: the inhibition-timing hypothesis. Brain Res. Rev. 53, 63-88. doi: 10.1016/j. brainresrev.2006.06.003

Lakatos, P., Karmos, G., Mehta, A. D., Ulbert, I., and Schroeder, C. E. (2008). Entrainment of neuronal oscillations as a mechanism of attentional selection. Science 320, 110-113. doi: 10.1126/science.1154735

Lakatos, P., Shah, A. S., Knuth, K. H., Ulbert, I., Karmos, G., and Schroeder, C. E. (2005). An oscillatory hierarchy controlling neuronal excitability and stimulus processing in the auditory cortex. J. Neurophysiol. 94, 1904-1911. doi: $10.1152 /$ jn. 00263.2005

Large, E. W., and Kolen, J. F. (1994). Resonance and the perception of musical meter. Conn. Sci. 6, 177-208. doi: 10.1080/09540099408915723

Makeig, S., Bell, A. J., Jung, T. P., and Sejnowski, T. J. (1996). "Independent component analysis of electroencephalographic data," in Advances in Neural Information Processing Systems, eds D. S. Touretzky, M. C. Mozer and M. E. Hasselmo (Cambridge, MA: MIT Press), 145-151.

Makeig, S., Debener, S., Onton, J., and Delorme, A. (2004). Mining eventrelated brain dynamics. Trends Cogn. Sci. 8, 204-210. doi: 10.1016/j.tics.2004. 03.008

Martínez-Cancino, R., Heng, J., Delorme, A., Kreutz-Delgado, K., Sotero, R. C., and Makeig, S. (2019). Measuring transient phase-amplitude coupling using local mutual information. Neuroimage 185, 361-378. doi: 10.1016/j. neuroimage.2018.10.034

Mathewson, K. E., Gratton, G., Fabiani, M., Beck, D. M., and Ro, T. (2009). To see or not to see: prestimulus $\alpha$ phase predicts visual awareness. J. Neurosci. 29 , 2725-2732. doi: 10.1523/JNEUROSCI.3963-08.2009

Mathewson, K. E., Lleras, A., Beck, D. M., Fabiani, M., Ro, T., and Gratton, G. (2011). Pulsed out of awareness: EEG $\alpha$ oscillations represent a pulsed-inhibition of ongoing cortical processing. Front. Psychol. 2:99. doi: 10.3389/fpsyg.2011.00099

Medaglia, J. D., Pasqualetti, F., Hamilton, R. H., Thompson-Schill, S. L., and Bassett, D. S. (2017). Brain and cognitive reserve: translation via network control theory. Neurosci. Biobehav. Rev. 75, 53-64. doi: 10.1016/j.neubiorev. 2017.01.016

Mercier, M. R., Molholm, S., Fiebelkorn, I. C., Butler, J. S., Schwartz, T. H., and Foxe, J. J. (2015). Neuro-oscillatory phase alignment drives speeded multisensory response times: an electro-corticographic investigation. J. Neurosci. 35, 8546-8557. doi: 10.1523/JNEUROSCI.4527-14.2015

Miller, K. J., Honey, C. J., Hermes, D., Rao, R. P., and Ojemann, J. G. (2014). Broadband changes in the cortical surface potential track activation of functionally diverse neuronal populations. Neuroimage 85, 711-720. doi: 10.1016/j.neuroimage.2013.08.070

Miller, K. J., Shenoy, P., Miller, J. W., Rao, R. P., and Ojemann, J. G. (2007). Real-time functional brain mapping using electrocorticography. Neuroimage 37, 504-507. doi: 10.1016/j.neuroimage.2007.05.029

Miller, K. J., Sorensen, L. B., Ojemann, J. G., and Den Nijs, M. (2009). Power-law scaling in the brain surface electric potential. PLoS Comput. Biol. 5:e1000609. doi: 10.1371/journal.pcbi.1000609

Müller-Dahlhaus, J. F. M., Orekhov, Y., Liu, Y., and Ziemann, U. (2008). Interindividual variability and age-dependency of motor cortical plasticity induced by paired associative stimulation. Exp. Brain Res. 187, 467-475. doi: 10.1007/s00221-008-1319-7

O’Reardon, J. P., Solvason, H. B., Janicak, P. G., Sampson, S., Isenberg, K. E., Nahas, Z., et al. (2007). Efficacy and safety of transcranial magnetic stimulation in the acute treatment of major depression: a multisite randomized controlled trial. Biol. Psychiatry 62, 1208-1216. doi: 10.1016/j.biopsych.2007. 01.018

Onton, J., Delorme, A., and Makeig, S. (2005). Frontal midline EEG dynamics during working memory. Neuroimage 27, 341-356. doi: 10.1016/j.neuroimage. 2005.04.014 
Onton, J., Westerfield, M., Townsend, J., and Makeig, S. (2006). Imaging human EEG dynamics using independent component analysis. Neurosci. Biobehav. Rev. 30, 808-822. doi: 10.1016/j.neubiorev.2006.06.007

Osipova, D., Hermes, D., and Jensen, O. (2008). $\gamma$ power is phase-locked to posterior $\alpha$ activity. PLoS One 3:e3990. doi: 10.1371/journal.pone.0003990

Perera, T., George, M. S., Grammer, G., Janicak, P. G., Pascual-Leone, A., and Wirecki, T. S. (2016). The clinical TMS society consensus review and treatment recommendations for TMS therapy for major depressive disorder. Brain Stimul. 9, 336-346. doi: 10.1016/j.brs.2016.03.010

Pfurtscheller, G., and Lopes da Silva, F. H. (1999). Event-related EEG/MEG synchronization and desynchronization: basic principles. Clin. Neurophysiol. 110, 1842-1857. doi: 10.1016/s1388-2457(99)00141-8

Pfurtscheller, G., and Neuper, C. (1997). Motor imagery activates primary sensorimotor area in humans. Neurosci. Lett. 239, 65-68. doi: 10.1016/s03043940(97)00889-6

Pfurtscheller, G., Neuper, C., Flotzinger, D., and Pregenzer, M. (1997). EEGbased discrimination between imagination of right and left hand movement. Electroencephalogr. Clin. Neurophysiol. 103, 642-651. doi: 10.1016/s00134694(97)00080-1

Pogarell, O., Koch, W., Pöpperl, G., Tatsch, K., Jakob, F., Zwanzger, P., et al. (2006). Striatal dopamine release after prefrontal repetitive transcranial magnetic stimulation in major depression: preliminary results of a dynamic [123I] IBZM SPECT study. J. Psychiatric Res. 40, 307-314. doi: 10.1016/j. jpsychires.2005.09.001

Reinhart, R. M., and Nguyen, J. A. (2019). Working memory revived in older adults by synchronizing rhythmic brain circuits. Nat. Neurosci. 22, 820-827. doi: 10.1038/s41593-019-0371-x

Ridding, M. C., and Rothwell, J. C. (2007). Is there a future for therapeutic use of transcranial magnetic stimulation? Nat. Rev. Neurosci. 8, 559-567. doi: $10.1038 / \mathrm{nrn} 2169$

Romei, V., Gross, J., and Thut, G. (2010). On the role of prestimulus $\alpha$ rhythms over occipito-parietal areas in visual input regulation: correlation or causation? J. Neurosci. 30, 8692-8697. doi: 10.1523/JNEUROSCI.0160-10.2010

Rossi, S., and Rossini, P. M. (2004). TMS in cognitive plasticity and the potential for rehabilitation. Trends Cogn. Sci. 8, 273-279. doi: 10.1016/s13646613(04)00115-9

Samaha, J., and Postle, B. R. (2015). The speed of $\alpha$-band oscillations predicts the temporal resolution of visual perception. Curr. Biol. 25, 2985-2990. doi: 10.1016/j.cub.2015.10.007

Sauseng, P., Klimesch, W., Gerloff, C., and Hummel, F. C. (2009a). Spontaneous locally restricted EEG $\alpha$ activity determines cortical excitability in the motor cortex. Neuropsychologia 47, 284-288. doi: 10.1016/j.neuropsychologia.2008. 07.021

Sauseng, P., Klimesch, W., Heise, K. F., Gruber, W. R., Holz, E., Karim, A. A., et al. (2009b). Brain oscillatory substrates of visual short-term memory capacity. Curr. Biol. 19, 1846-1852. doi: 10.1016/j.cub.2009.08.062

Scheeringa, R., Mazaheri, A., Bojak, I., Norris, D. G., and Kleinschmidt, A. (2011). Modulation of visually evoked cortical FMRI responses by phase of ongoing occipital $\alpha$ oscillations. J. Neurosci. 31, 3813-3820. doi: 10.1523/JNEUROSCI. 4697-10.2011

Schmidt, S. L., Iyengar, A. K., Foulser, A. A., Boyle, M. R., and Fröhlich, F. (2014). Endogenous cortical oscillations constrain neuromodulation by weak electric fields. Brain Stimul. 7, 878-889. doi: 10.1016/j.brs.2014.07.033

Schnitzler, A., and Gross, J. (2005). Normal and pathological oscillatory communication in the brain. Nat. Rev. Neurosci. 6, 285-296. doi: 10.1038/nrn1650

Schroeder, C. E., and Lakatos, P. (2009). Low-frequency neuronal oscillations as instruments of sensory selection. Trends Neurosci. 32, 9-18. doi: 10.1016/j.tins. 2008.09.012

Shapiro, K. L., Hanslmayr, S., Enns, J. T., and Lleras, A. (2017). $\alpha, \beta$ : the rhythm of the attentional blink. Psychon. Bull. Rev. 1824, 1862-1869. doi: 10.3758/s13423017-1257-0

Spaak, E., de Lange, F. P., and Jensen, O. (2014). Local entrainment of $\alpha$ oscillations by visual stimuli causes cyclic modulation of perception. J. Neurosci. 34, 3536-3544. doi: 10.1523/JNEUROSCI.4385-13.2014

Strafella, A. P., Paus, T., Barrett, J., and Dagher, A. (2001). Repetitive transcranial magnetic stimulation of the human prefrontal cortex induces dopamine release in the caudate nucleus. J. Neurosci. 21, RC157-RC157. doi: 10.1523/JNEUROSCI.21-15-j0003.2001
Thut, G., Bergmann, T. O., Fröhlich, F., Soekadar, S. R., Brittain, J. S., ValeroCabré, A., et al. (2017). Guiding transcranial brain stimulation by EEG/MEG to interact with ongoing brain activity and associated functions: a position paper. Clin. Neurophysiol. 128, 843-857. doi: 10.1016/j.clinph.2017.01.003

Thut, G., and Pascual-Leone, A. (2010). A review of combined TMS-EEG studies to characterize lasting effects of repetitive TMS and assess their usefulness in cognitive and clinical neuroscience. Brain Topogr. 22, 219-232. doi: 10.1007/s10548-009-0115-4

Thut, G., Veniero, D., Romei, V., Miniussi, C., Schyns, P., and Gross, J. (2011). Rhythmic TMS causes local entrainment of natural oscillatory signatures. Curr. Biol. 21, 1176-1185. doi: 10.1016/j.cub.2011.05.049

Tik, M., Hoffmann, A., Sladky, R., Tomova, L., Hummer, A., Navarro de Lara, L., et al. (2017). Towards understanding rTMS mechanism of action: stimulation of the DLPFC causes network-specific increase in functional connectivity. Neuroimage 162, 289-296. doi: 10.1016/j.neuroimage.2017.09.022

Uhlhaas, P. J., and Singer, W. (2010). Abnormal neural oscillations and synchrony in schizophrenia. Nat. Rev. Neurosci. 11, 100-113. doi: 10.1038/ $\operatorname{nrn} 2774$

van Dijk, H., Schoffelen, J. M., Oostenveld, R., and Jensen, O. (2008). Prestimulus oscillatory activity in the $\alpha$ band predicts visual discrimination ability. J. Neurosci. 28, 1816-1823. doi: 10.1523/JNEUROSCI.1853-07.2008

Vanhatalo, S., Palva, J. M., Holmes, M. D., Miller, J. W., Voipio, J., and Kaila, K. (2004). Infraslow oscillations modulate excitability and interictal epileptic activity in the human cortex during sleep. Proc. Natl. Acad. Sci. U S A 101, 5053-5057. doi: 10.1073/pnas.0305375101

Varela, F. J., Toro, A., John, E. R., and Schwartz, E. L. (1981). Perceptual framing and cortical $\alpha$ rhythm. Neuropsychologia 19, 675-686. doi: 10.1016/00283932(81)90005-1

Veniero, D., Brignani, D., Thut, G., and Miniussi, C. (2011). $\alpha$-generation as basic response-signature to transcranial magnetic stimulation (TMS) targeting the human resting motor cortex: a TMS/EEG co-registration study. Psychophysiology 48, 1381-1389. doi: 10.1111/j.1469-8986.2011.01218.x

Vöröslakos, M., Takeuchi, Y., Brinyiczki, K., Zombori, T., Oliva, A., FernándezRuiz, A., et al. (2018). Direct effects of transcranial electric stimulation on brain circuits in rats and humans. Nat. Commun. 9:483. doi: 10.1038/s41467-01802928-3

Voytek, B., Canolty, R. T., Shestyuk, A., Crone, N., Parvizi, J., and Knight, R. T. (2010). Shifts in $\gamma$ phase-amplitude coupling frequency from theta to a over posterior cortex during visual tasks. Front. Hum. Neurosci. 4:191. doi: 10.3389/fnhum.2010.00191

Voytek, B., D’Esposito, M., Crone, N., and Knight, R. T. (2013). A method for event-related phase/amplitude coupling. Neuroimage 64, 416-424. doi: 10.1016/j.neuroimage.2012.09.023

Zarkowski, P., Shin, C. J., Dang, T., Russo, J., and Avery, D. (2006). EEG and the variance of motor evoked potential amplitude. Clin. EEG Neurosci. 37, 247-251. doi: 10.1177/155005940603700316

Zauner, A., Fellinger, R., Gross, J., Hanslmayr, S., Shapiro, K., Gruber, W., et al. (2012). \& entrainment is responsible for the attentional blink phenomenon. Neuroimage 63, 674-686. doi: 10.1016/j.neuroimage.2012. 06.075

Ziemann, U., and Siebner, H. R. (2015). Inter-subject and inter-session variability of plasticity induction by non-invasive brain stimulation: boon or bane? Brain Stimul. 8, 662-663. doi: 10.1016/j.brs.2015.01.409

Zrenner, C., Desideri, D., Belardinelli, P., and Ziemann, U. (2018). Real-time EEG-defined excitability states determine efficacy of TMS-induced plasticity in human motor cortex. Brain Stimul. 11, 374-389. doi: 10.1016/j.brs.2017. 11.016

Conflict of Interest Statement: The authors declare that the research was conducted in the absence of any commercial or financial relationships that could be construed as a potential conflict of interest.

Copyright (C) 2019 Wagner, Makeig, Hoopes and Gola. This is an open-access article distributed under the terms of the Creative Commons Attribution License (CC BY). The use, distribution or reproduction in other forums is permitted, provided the original author(s) and the copyright owner(s) are credited and that the original publication in this journal is cited, in accordance with accepted academic practice. No use, distribution or reproduction is permitted which does not comply with these terms. 